\title{
Artery of Adamkiewicz on 256-slice Multislice CT
}

\author{
Shu Yoshihara ${ }^{1}$, Masaki Kamiya ${ }^{2}$, Masaaki Naito ${ }^{1}$ and Masaki Matsunaga ${ }^{3}$
}

Key words: artery of Adamkiewicz, multislice computed tomography, aortic dissection

(Intern Med 53: 329-330, 2014)

(DOI: 10.2169/internalmedicine.53.1568)

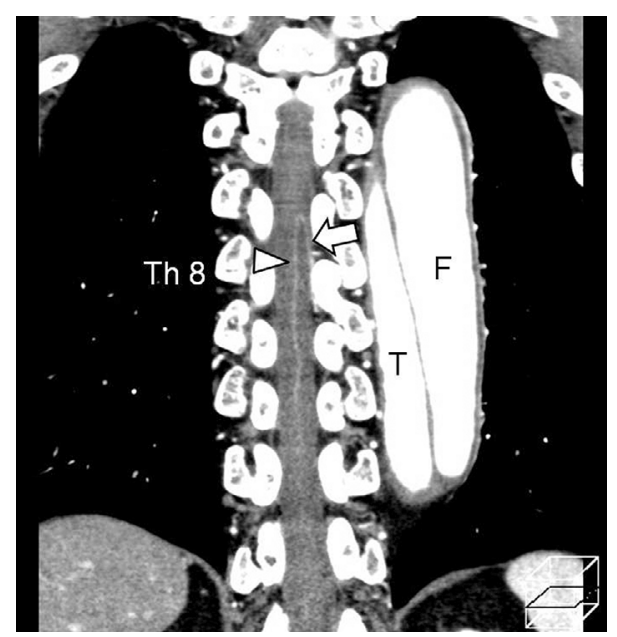

Picture 1.

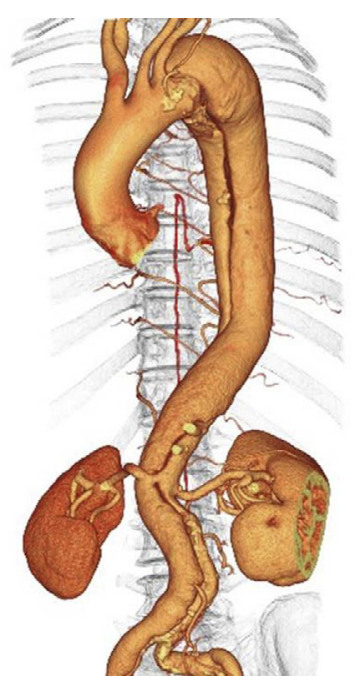

Picture 3.

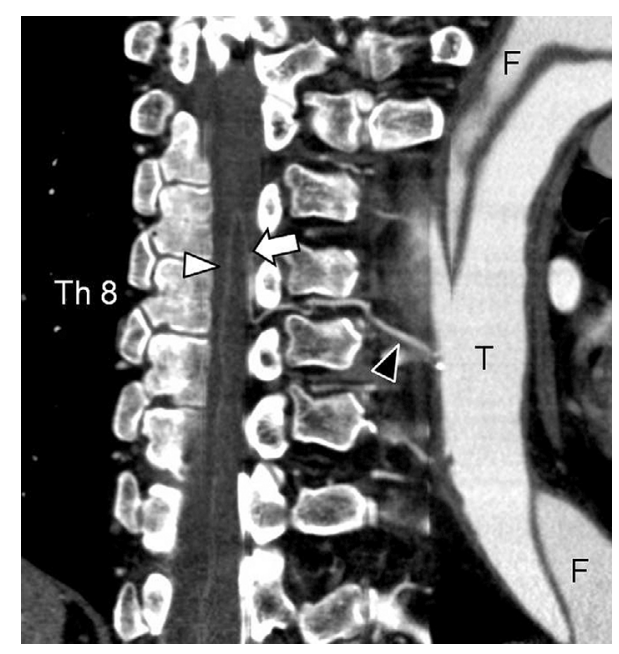

Picture 2.

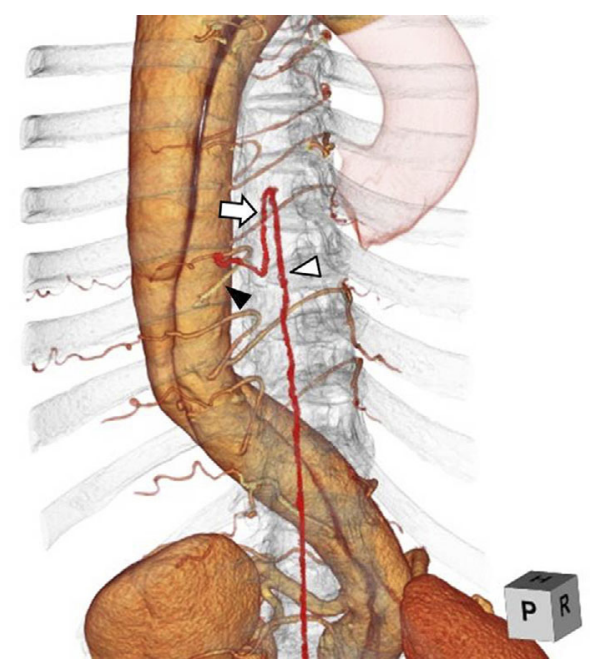

Picture 4.
A 73-year-old man with chronic Stanford type B aortic dissection with a maximum diameter of $58 \mathrm{~mm}$ and a com- pletely patent false lumen was scheduled for surgical repair. We performed computed tomography angiography (CTA) as

${ }^{1}$ Department of Diagnostic Radiology, Iwata City Hospital, Japan, ${ }^{2}$ Department of Radiological Technology, Iwata City Hospital, Japan and ${ }^{3}$ Department of Cardiology, Iwata City Hospital, Japan

Received for publication August 14, 2013; Accepted for publication September 2, 2013

Correspondence to Dr. Shu Yoshihara, shuy@hospital.iwata.shizuoka.jp 
a preoperative evaluation to visualize the artery of Adamkiewicz (AKA) using a 256-slice multislice CT scanner (Brilliance iCT, Philips Healthcare, Best, The Netherlands). A coronal multiplanar-reformatted image showed a hairpin curved AKA (arrow) joining the anterior spinal artery (arrowhead) (Picture 1, T: true lumen, F: false lumen, Th8: 8th thoracic vertebra). A curved multiplanar-reformatted image (Picture 2, T: true lumen, F: false lumen, Th8: 8th thoracic vertebra) and 3D volume-rendered images (Picture 3: anterior view, Picture 4: right posterosuperior view) clearly visu- alized the continuity between the AKA and the true lumen of the aorta through the left 8th intercostal artery (black arrowhead). The patient underwent surgery without spinal cord complications. Depicting the AKA is important for surgical planning in cases of a thoracic descending aorta in order to prevent postoperative paraplegia. CTA is a useful method for evaluating the AKA.

The authors state that they have no Conflict of Interest (COI).

(C) 2014 The Japanese Society of Internal Medicine http://www.naika.or.jp/imonline/index.html 